\title{
One-dimensional lattice of oscillators coupled through power-law interactions: Continuum limit and dynamics of spatial Fourier modes
}

\author{
Shamik Gupta, ${ }^{1}$ Max Potters, ${ }^{1,2}$ and Stefano Ruffo ${ }^{1,3}$ \\ ${ }^{1}$ Laboratoire de Physique de l'École Normale Supérieure de Lyon, Université de Lyon, CNRS, 46 Allée d'Italie, 69364 Lyon cédex 07, France \\ ${ }^{2}$ Delft Center for Systems and Control, Technical University Delft, Mekelweg 2, 2628 CD Delft, The Netherlands \\ ${ }^{3}$ Dipartimento di Energetica "Sergio Stecco" and CSDC, Università di Firenze, CNISM and INFN, via S. Marta 3, 50139 Firenze, Italy
}

(Received 3 March 2012; published 4 June 2012)

\begin{abstract}
We study synchronization in a system of phase-only oscillators residing on the sites of a one-dimensional periodic lattice. The oscillators interact with a strength that decays as a power law of the separation along the lattice length and is normalized by a size-dependent constant. The exponent $\alpha$ of the power law is taken in the range $0 \leqslant \alpha<1$. The oscillator frequency distribution is symmetric about its mean (taken to be zero) and is nonincreasing on $[0, \infty)$. In the continuum limit, the local density of oscillators evolves in time following the continuity equation that expresses the conservation of the number of oscillators of each frequency under the dynamics. This equation admits as a stationary solution the unsynchronized state uniform both in phase and over the space of the lattice. We perform a linear stability analysis of this state to show that when it is unstable, different spatial Fourier modes of fluctuations have different stability thresholds beyond which they grow exponentially in time with rates that depend on the Fourier modes. However, numerical simulations show that at long times all the nonzero Fourier modes decay in time, while only the zero Fourier mode (i.e., the "mean-field" mode) grows in time, thereby dominating the instability process and driving the system to a synchronized state. Our theoretical analysis is supported by extensive numerical simulations.
\end{abstract}

DOI: 10.1103/PhysRevE.85.066201

PACS number(s): 05.45.Xt

\section{INTRODUCTION: THE MODEL}

A large collection of coupled oscillating components of different natural frequencies getting spontaneously synchronized to oscillate at a common frequency is a phenomenon commonly observed in a variety of biological and physical systems, e.g., yeast cell suspensions [1,2], networks of pacemaker cells in the heart [3,4], groups of flashing fireflies [5,6], arrays of superconducting Josephson junctions $[7,8]$, and many others [9].

The Kuramoto model provides a simple setting to investigate the physics of synchronization [10-12]. The model considers a large population of phase-only oscillators of distributed natural frequencies. The oscillators are globally coupled with coupling strengths that are equal and independent of their spatial distribution. The governing dynamical equations are

$$
\frac{d \theta_{i}}{d t}=\omega_{i}+\frac{K}{N} \sum_{j=1, j \neq i}^{N} \sin \left(\theta_{j}-\theta_{i}\right)
$$

for $i=1,2, \ldots, N \gg 1$. Here $\theta_{i}$ is a periodic variable of period $2 \pi$ that denotes the phase of the $i$ th oscillator whose natural frequency is given by $\omega_{i} \in R$. The parameter $K \geqslant 0$ denotes the coupling strength. The frequencies are distributed according to a probability density $g(\omega)$, assumed to be onehumped and symmetric about its mean. It is known that above a critical coupling strength $K_{c}=2 /(\pi g(0))$, the stationary state that the system attains at long times is a synchronized state. For $K<K_{c}$, however, there is no synchronization at long times, and each oscillator keeps running at its own natural frequency.

Although the Kuramoto model has been studied extensively in recent years, much less is known about the case in which the oscillators are coupled with a strength that is a function of their spatial distribution, e.g., an inverse power law in the separation.
Such a form of interaction is relevant for many situations, for example, in the study of long-range synchrony in a network of coupled neurons [13] or in modeling flashing fireflies where the intensity of light signals carrying information from one firefly to another falls off in the three-dimensional space as the inverse of square of the distance from the source.

A simple modification of the Kuramoto model (1) to include a power-law interaction is to consider a one-dimensional periodic lattice of $N$ sites, with each site containing one oscillator, and with the oscillators interacting with one another with a strength that decays as a power law of the separation along the lattice length [14]. Consequently the governing equations are modified from (1) to

$$
\frac{d \theta_{i}}{d t}=\omega_{i}+\frac{K}{\widetilde{N}} \sum_{j=1, j \neq i}^{N} \frac{\sin \left(\theta_{j}-\theta_{i}\right)}{\left|x_{j}-x_{i}\right|^{\alpha}} ; \quad \alpha \geqslant 0 .
$$

Here $x_{j}=j \epsilon$ is the coordinate of the $j$ th site on the lattice, where $\epsilon$ is the lattice constant. The constant $\widetilde{N}=\sum_{j=1}^{N} \mid x_{j}-$ $\left.x_{i}\right|^{-\alpha} \forall i$ is a size-dependent normalization. Being on a periodic lattice, we adopt the closest distance convention:

$$
\left|x_{j}-x_{i}\right|=\min \left(\left|x_{j}-x_{i}\right|, 1-\left|x_{j}-x_{i}\right|\right),
$$

where the total length $N \epsilon$ of the lattice has been taken to equal unity without loss of generality. From Eq. (2), we see that for $\alpha<1$, the cumulative interaction of one oscillator with all the remaining oscillators with aligned phases would diverge in the continuum limit $N \rightarrow \infty$ in the absence of the normalization $\widetilde{N}$, which explains its inclusion [14,15]. Note that the case $\alpha=$ 0 of the model corresponds to the usual Kuramoto model (1). Let us mention that a model with space-dependent phases as in Eq. (2), but with a different form of coupling between the oscillators, has recently been studied in Ref. [16]. 
A previous study of the model (2) addressed by numerical simulations the issue of synchronization, in particular, the conditions for its emergence at long times as a function of $\alpha$ for a fixed value of $K$ [14]. It was reported that a critical value $\alpha_{c}=2$ exists, such that only when $\alpha<\alpha_{c}$ does the system in the limit $N \rightarrow \infty$ synchronize at a finite value of $K$. For $\alpha>\alpha_{c}$, there is no finite value of $K$ at which synchronization occurs. A recent theoretical study, however, predicted the value $3 / 2$ for $\alpha_{c}$ [17]. In Ref. [18] the model was studied without the normalization $\widetilde{N}$, and for finite values of $N$. In this work mainly numerical simulations were employed to investigate the dependence of $N$ on $\alpha$ and $K$ that leads to synchronization.

In this paper we consider the model (2) with values of $\alpha$ in the range $0 \leqslant \alpha<1$. We take the frequency distribution $g(\omega)$ to be symmetric about its mean (taken to be zero) and nonincreasing on $[0, \infty)$. We analyze the model (2) in the continuum limit $N \rightarrow \infty$, when the lattice is replaced by a continuous line characterized by the spatial coordinate $x \in[0,1]$. Since in this limit each infinitesimal element $d x$ contains a diverging number of oscillators, it is natural to define a local density of oscillators $\rho(\theta ; \omega, x, t) d x$ such that of all oscillators with natural frequency $\omega$ that are located between $x$ and $x+d x$ at time $t$, the fraction $\rho(\theta ; \omega, x, t) d \theta d x$ have their phase between $\theta$ and $\theta+d \theta$. Because the dynamics conserves the total number of oscillators with frequency $\omega$, the time evolution of $\rho(\theta ; \omega, x, t)$ is given by the continuity equation. In this equation the local densities at different spatial locations appear coupled due to the interaction between the oscillators.

The unsynchronized state, uniform both in the phase $\theta$ and in the spatial coordinate $x$, solves the continuity equation in the stationary state. By performing a linear stability analysis of such a state, we show that when it is unstable, different spatial Fourier modes of fluctuations have different stability thresholds beyond which they grow exponentially in time with different rates. However, numerical simulations show that at long times, all the nonzero Fourier modes decay in time, while the zero mode (the "mean-field" mode) grows in time and dominates the instability process, thereby driving the system to a synchronized state. Such a long-time dominance of the mean-field mode in characterizing dynamic instability has been observed in systems with power-law interactions evolving under a deterministic Hamiltonian dynamics [19]. The present study demonstrates this dominance for the dissipative dynamics of the model (2). Our theoretical predictions for the growth rates of the unstable modes are corroborated by numerical simulations.

The outline of this paper is as follows. In the following section, we consider the model (2) in the continuum limit $N \rightarrow \infty$ and analyze the linear stability of the unsynchronized state. In the region in which it is unstable, we derive analytic expressions for the stability thresholds and the growth rates of the various Fourier modes of fluctuations. In Sec. III we test our theoretical predictions for the growth rates by performing numerical simulations and illustrate the dominance of the mean-field mode in characterizing the long-time instability of the unsynchronized state. In simulations, we employ a fast numerical algorithm to compute the interaction among the oscillators, the details of which are relegated to the Appendix. In the final section, we draw our conclusions.

\section{CONTINUUM LIMIT ANALYSIS}

In this section, we consider the model (2) in the continuum limit $N \rightarrow \infty$, and investigate in detail the issue of linear stability of the unsynchronized state. Finite- $N$ effects are known to be quite subtle and difficult to tackle even for the usual Kuramoto model [11,12], so we do not attempt a finite- $N$ analysis of the model (2) in this work.

In the continuum limit, the lattice of the system (2) is densely filled with sites. Let the continuous variable $x \in[0,1]$ stand for the spatial coordinate along the lattice length. Now that each infinitesimal element $d x$ contains a diverging number of oscillators, we define a local density of oscillators $\rho(\theta ; \omega, x, t) d x$ such that of all oscillators with natural frequency $\omega$ that are located between $x$ and $x+d x$ at time $t$, the fraction $\rho(\theta ; \omega, x, t) d \theta d x$ have their phase between $\theta$ and $\theta+d \theta$. This density is non-negative, $2 \pi$ periodic in $\theta$, and obeys the normalization

$$
\int_{0}^{2 \pi} d \theta \rho(\theta ; \omega, x, t)=1 .
$$

The equations of motion (2) become

$$
\begin{aligned}
\frac{\partial \theta(\omega, x, t)}{\partial t}= & \omega+\kappa(\alpha) K \int d \theta^{\prime} d \omega^{\prime} \\
& \times d x^{\prime} \frac{\sin \left(\theta^{\prime}-\theta\right)}{\left|x^{\prime}-x\right|^{\alpha}} \rho\left(\theta^{\prime} ; \omega^{\prime}, x^{\prime}, t\right) g\left(\omega^{\prime}\right),
\end{aligned}
$$

where $\kappa(\alpha)$ is such that $N / \widetilde{N} \rightarrow \kappa(\alpha)$ as $N \rightarrow \infty$, and therefore one has

$$
\kappa(\alpha)^{-1}=\int_{x-1 / 2}^{x+1 / 2} \frac{d x^{\prime}}{\left|x^{\prime}-x\right|^{\alpha}} .
$$

Since we consider $\alpha$ in the range $0 \leqslant \alpha<1$, the above integral is convergent. The number of oscillators with frequency $\omega$ is conserved by the dynamics, so that the time evolution of $\rho(\theta ; \omega, x, t)$ follows the continuity equation

$$
\frac{\partial \rho}{\partial t}=-\frac{\partial}{\partial \theta}\left(\rho \frac{\partial \theta}{\partial t}\right)
$$

where $\frac{\partial \theta}{\partial t}$ is given by Eq. (5).

Next, consider the unsynchronized state uniform both in the phase $\theta$ and in the spatial coordinate $x$ :

$$
\rho_{0}(\theta ; \omega, x, t)=\frac{1}{2 \pi} \text {. }
$$

Such a state is evidently a stationary solution of the time evolution (7). To study its linear stability under the dynamics, consider adding small fluctuations, so that

$$
\rho(\theta ; \omega, x, t)=\frac{1}{2 \pi}+\delta \rho(\theta ; \omega, x, t) ; \quad \delta \rho(\theta ; \omega, x, t) \ll 1 .
$$

Substituting into Eq. (7) and keeping terms to linear order in $\delta \rho$, we find that $\delta \rho$ satisfies

$$
\begin{aligned}
\frac{\partial \delta \rho(\theta ; \omega, x, t)}{\partial t}= & -\omega \frac{\partial \delta \rho(\theta ; \omega, x, t)}{\partial \theta}+\frac{\kappa(\alpha) K}{2 \pi} \int d \theta^{\prime} d \omega^{\prime} \\
& \times d x^{\prime} \frac{\cos \left(\theta^{\prime}-\theta\right)}{\left|x^{\prime}-x\right|^{\alpha}} \delta \rho\left(\theta^{\prime} ; \omega^{\prime}, x^{\prime}, t\right) g\left(\omega^{\prime}\right) .
\end{aligned}
$$




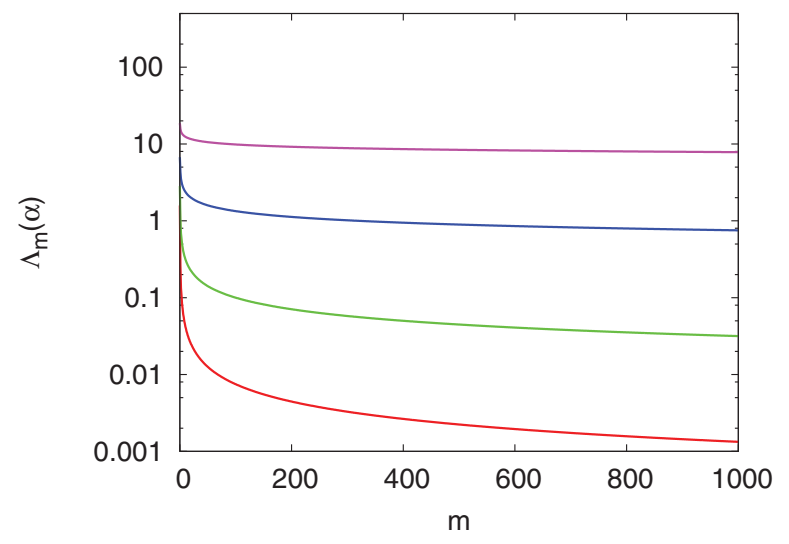

FIG. 1. (Color online) $\Lambda_{m}(\alpha)$ as a function of $m$, obtained by numerically evaluating the integral in Eq. (15). The different curves correspond to different $\alpha$ values given by $\alpha=0.25,0.5,0.75,0.9$ (bottom to top).

Expressing $\delta \rho$ in terms of its Fourier series with respect to the periodic variable $\theta$ as

$$
\delta \rho(\theta ; \omega, x, t)=\sum_{k=-\infty}^{\infty} \widehat{\delta \rho}_{k}(\omega, x, t) e^{i k \theta},
$$

we find from Eq. (10) that only the modes $k= \pm 1$ are affected by the coupling between the oscillators, and that $\widehat{\delta \rho_{ \pm 1}}$ satisfies

$$
\begin{aligned}
\frac{\partial \widehat{\delta \rho}_{ \pm 1}(\omega, x, t)}{\partial t}= & \mp i \omega \widehat{\delta \rho}_{ \pm 1}(\omega, x, t)+\frac{\kappa(\alpha) K}{2} \\
& \times \int d \omega^{\prime} d x^{\prime} \frac{\widehat{\delta \rho}_{ \pm 1}\left(\omega^{\prime}, x^{\prime}, t\right)}{\left|x^{\prime}-x\right|^{\alpha}} g\left(\omega^{\prime}\right) .
\end{aligned}
$$

One thus gets a set of equations for each position $x$, all coupled together by the second term on the right.

Since we have a periodic lattice, it is natural to consider the spatial Fourier series of $\widehat{\delta \rho_{ \pm 1}}(\omega, x, t)$ :

$$
\widehat{\delta \rho}_{ \pm 1}(\omega, x, t)=\sum_{m=-\infty}^{\infty} \overline{\delta \rho}_{ \pm 1, m}(\omega, t) e^{i 2 \pi m x}
$$

On substituting in Eq. (12), we get

$$
\begin{aligned}
\frac{\partial \overline{\delta \rho}_{ \pm 1, m}(\omega, t)}{\partial t}= & \mp i \omega \overline{\delta \rho}_{ \pm 1, m}(\omega, t)+\frac{\kappa(\alpha) K \Lambda_{m}(\alpha)}{2} \\
& \times \int d \omega^{\prime} \overline{\delta \rho}_{ \pm 1, m}\left(\omega^{\prime}, t\right) g\left(\omega^{\prime}\right),
\end{aligned}
$$

where

$$
\Lambda_{m}(\alpha)=\int_{x-1 / 2}^{x+1 / 2} d x^{\prime} \frac{e^{i 2 \pi m\left(x^{\prime}-x\right)}}{\left|x^{\prime}-x\right|^{\alpha}}
$$

Note that $\Lambda_{m}(\alpha)=\Lambda_{-m}(\alpha)$. We have checked numerically that $\Lambda_{m}(\alpha) \geqslant 0$, and that it is a monotonically decreasing function of $m$; see Fig. 1. These properties can also be proved analytically [20]. It is also easy to prove that $\Lambda_{m}(\alpha) \rightarrow 0$ as $m \rightarrow \infty$. Defining $w \equiv m y$, we get

$$
\Lambda_{m}(\alpha)=\frac{2}{m^{1-\alpha}} \int_{0}^{m / 2} d w \frac{\cos (2 \pi w)}{w^{\alpha}} .
$$

In the limit $m \rightarrow \infty$, the integral evaluates to a finite constant equal to $(2 \pi)^{\alpha-1} \sin (\pi \alpha / 2) \Gamma(1-\alpha)$, while the prefactor tends to zero, yielding

$$
\lim _{m \rightarrow \infty} \Lambda_{m}(\alpha)=0
$$

Following [11], we consider the right-hand side of Eq. (14) to define a linear operator $A$ as

$$
\begin{aligned}
A \overline{\delta \rho}_{ \pm 1, m}(\omega, t)= & \mp i \omega \overline{\delta \rho}_{ \pm 1, m}(\omega, t)+\frac{\kappa(\alpha) K \Lambda_{m}(\alpha)}{2} \\
& \times \int d \omega^{\prime} \overline{\delta \rho}_{ \pm 1, m}\left(\omega^{\prime}, t\right) g\left(\omega^{\prime}\right) .
\end{aligned}
$$

Then, in terms of the eigenvalues $\lambda_{m}$ of the operator $A$, Eq. (14) gives

$$
\overline{\delta \rho}_{ \pm 1, m}(\omega, t)=\tilde{\delta \rho_{ \pm 1, m}}\left(\omega, \lambda_{m}\right) e^{\lambda_{m} t}
$$

with $\tilde{\delta \rho_{ \pm 1, m}}$ satisfying

$$
\begin{aligned}
& \tilde{\delta \rho}_{ \pm 1, m}\left(\omega, \lambda_{m}\right) \\
& \quad=\frac{\kappa(\alpha) K \Lambda_{m}(\alpha)}{2\left(\lambda_{m} \pm i \omega\right)} \int d \omega^{\prime} \tilde{\delta \rho}_{ \pm 1, m}\left(\omega^{\prime}, \lambda_{m}\right) g\left(\omega^{\prime}\right) .
\end{aligned}
$$

Multiplying both sides of Eq. (20) by $g(\omega)$, and then integrating with respect to $\omega$, one gets

$$
I_{ \pm, m}\left(1-J_{ \pm, m}\right)=0
$$

where

$$
\begin{gathered}
I_{ \pm, m}=\int_{-\infty}^{\infty} d \omega \tilde{\delta \rho_{ \pm 1, m}}\left(\omega, \lambda_{m}\right) g(\omega), \\
J_{ \pm, m}=\frac{\kappa(\alpha) K \Lambda_{m}(\alpha)}{2} \int_{-\infty}^{\infty} d \omega \frac{g(\omega)}{\left(\lambda_{m} \pm i \omega\right)} .
\end{gathered}
$$

Since $I_{ \pm, m} \neq 0$, Eq. (21) gives the dispersion relation

$$
J_{ \pm, m}=1 .
$$

Let us consider $g(\omega)$ to be symmetric about its mean. We may assume a zero mean without loss of generality (one can easily achieve this by going into a rotating frame of reference). Moreover, we take $g(\omega)$ to be nonincreasing on $[0, \infty)$, i.e., $g(\omega) \geqslant g(\nu)$ whenever $\omega \leqslant \nu$. Examples include common frequency distributions of interest like the Gaussian and the Lorentzian distributions. One can prove by using Theorem 2 in Ref. [21] that for such $g(\omega)$ 's, Eq. (24) has at most one solution for $\lambda_{m}$, and if it exists, it is necessarily real. Then Eq. (24) gives

$$
\kappa(\alpha) K \Lambda_{m}(\alpha) \int_{0}^{\infty} d \omega \frac{\lambda_{m}}{\lambda_{m}^{2}+\omega^{2}} g(\omega)=1 .
$$

The above equation implies that $\lambda_{m} \geqslant 0$, as otherwise the left-hand side is negative. Since the $m$ th spatial mode of fluctuations $\overline{\delta \rho}_{ \pm 1, m}$ has the time dependence $\overline{\delta \rho}_{ \pm 1, m} \sim e^{\lambda_{m} t}$ [Eq. (19)], it follows that this mode is either linearly neutrally stable corresponding to $\lambda_{m}=0$, or that it is linearly unstable corresponding to $\lambda_{m}>0$. The borderline between these two behaviors is achieved at the critical coupling $K_{c}^{(m)}$, obtained by taking the limit $\lambda_{m} \rightarrow 0^{+}$in Eq. (25); we get

$$
K_{c}^{(m)}=\frac{2}{\kappa(\alpha) \Lambda_{m}(\alpha) \pi g(0)} .
$$


Combining the last equation with Eq. (25), the growth rate $\lambda_{m}>0$ for $K>K_{c}^{(m)}$ is given by

$$
\frac{2 K}{\pi g(0) K_{c}^{(m)}} \int_{0}^{\infty} d \omega \frac{\lambda_{m}}{\lambda_{m}^{2}+\omega^{2}} g(\omega)=1 .
$$

With $\Lambda_{m}(\alpha)=\Lambda_{-m}(\alpha)$, it follows that the Fourier modes $m$ and $-m$ have the same critical thresholds and the same growth rates. Since $\Lambda_{|m|}(\alpha)$ decreases on increasing $|m|$, we conclude that

$$
K_{c}^{(0)}<K_{c}^{(1)}<K_{c}^{(2)}<\cdots .
$$

The unsynchronized state (8) is linearly stable if all the spatial modes of fluctuations decay in time; using Eq. (28), this is achieved when $K<K_{c}^{(0)}$. On the other hand, it becomes unstable for $K>K_{c}^{(0)}$.

For $\alpha=0$, when our model (2) reduces to the Kuramoto model (1), Eqs. (6) and (15) give $\kappa(\alpha) \Lambda_{m}(\alpha)=\delta_{m, 0}$. In this limit the oscillators are globally coupled with equal coupling strengths, and therefore, it is physical to talk of only the zero Fourier mode. This mode has the critical coupling given by Eq. (26) to be $K_{c}^{(0)}=2 / \pi g(0)$, such that it is linearly unstable for higher values of $K$; this matches with known results for the Kuramoto model $[11,12]$. For finite values of $K$, all the other modes are linearly neutrally stable because of diverging critical thresholds.

In order to have some representative numbers for the critical thresholds $K_{c}^{(m)}$ and for comparison with numerical simulations reported in the next section, let us choose a Gaussian $g(\omega)$ with zero mean and unit variance:

$$
g(\omega)=\frac{1}{\sqrt{2 \pi}} e^{-\omega^{2} / 2} .
$$

Then, using Eq. (27), we see that for $K>K_{c}^{(m)}$, the growth rate $\lambda_{m}>0$ of the $m$ th mode satisfies

$$
\frac{K}{K_{c}^{(m)}} e^{\lambda_{m}^{2} / 2} \operatorname{Erfc}\left[\frac{\lambda_{m}}{\sqrt{2}}\right]=1,
$$

where $\operatorname{Erfc}[x]$ is the complementary error function: $\operatorname{Erfc}[x]=$ $\frac{2}{\sqrt{\pi}} \int_{x}^{\infty} e^{-t^{2}} d t$. In obtaining Eq. (30), we have used the result $\int_{-\infty}^{\infty} d p \frac{e^{-\beta p^{2} / 2}}{p^{2}+a^{2}}=\frac{\pi}{a} e^{\beta a^{2} / 2} \operatorname{Erfc}\left[\sqrt{\frac{\beta}{2}} a\right]$.

\section{COMPARISON WITH NUMERICAL SIMULATIONS}

Here we test the theoretical predictions of the preceding section by comparing them with results from numerical simulations. A standard procedure for simulations is to integrate the equations of motion (2). However, note that the equations involve computing at every integration step a sum over $N$ terms for each of the $N$ oscillators and therefore require a total computation time that would scale with $N$ as $N^{2}$. In the Appendix, we show that the equations of motion can be transformed into a convenient form which when integrated requires at every integration step a computation time that scales with $N$ as $N \ln N$. We chose this alternative form of the equations of motion and integrated them using a fourth-order Runge-Kutta algorithm with time step equal to 0.01 . The initial state of the system was chosen to have each oscillator phase $\theta$ uniformly distributed in $[-\pi, \pi]$ and frequency $\omega$ sampled from the Gaussian distribution (29), independently
TABLE I. $K_{c}^{(m)} ; m=1,2, \ldots, 5$ as a function of $\alpha$ for the frequency distribution (29). In all cases, $K_{c}^{(0)} \approx 1.59577$.

\begin{tabular}{lrrrrr}
\hline \hline$\alpha$ & \multicolumn{1}{c}{$K_{c}^{(1)}$} & \multicolumn{1}{c}{$K_{c}^{(2)}$} & \multicolumn{1}{c}{$K_{c}^{(3)}$} & \multicolumn{1}{c}{$K_{c}^{(4)}$} & \multicolumn{1}{c}{$K_{c}^{(5)}$} \\
\hline 0.05 & 52.96519 & 130.12769 & 165.38633 & 240.09713 & 274.75919 \\
0.1 & 25.89784 & 59.98356 & 76.10483 & 107.39521 & 122.97195 \\
0.5 & 4.26696 & 6.53664 & 7.71516 & 9.10681 & 10.02918 \\
0.9 & 1.88898 & 2.04629 & 2.12267 & 2.18907 & 2.23565 \\
0.95 & 1.73443 & 1.80472 & 1.83837 & 1.86673 & 1.88661 \\
\hline \hline
\end{tabular}

of all the other oscillators. Thus, initially, the system is in an unsynchronized state. We report here simulation results for $N=2^{14}$.

We specifically compare the theoretical and simulation results for the growth rates $\lambda_{m}$. From Eq. (30) these rates depend on the critical thresholds $K_{c}^{(m)}$ 's, and, thus, their comparison would indirectly provide a test of the theoretical predictions for $K_{c}^{(m)}$ 's given by Eqs. (26) and (29) as

$$
K_{c}^{(m)}=\frac{2 \sqrt{2}}{\sqrt{\pi} \kappa(\alpha) \Lambda_{m}(\alpha)} .
$$

Using the above equation, we show in Table I the dependence of $K_{c}^{(m)}$ 's for $m=1,2,3,4,5$, as a function of $\alpha$; note that $K_{c}^{(0)}$ is independent of $\alpha$. From the table, we see that the thresholds for the nonzero Fourier modes get larger and further apart when $\alpha$ is close to zero. On the contrary, these thresholds approach the threshold for the zero mode when $\alpha$ has a value close to unity. From this observation, one may guess that in the limit of $\alpha$ approaching zero, all thresholds excepting the one for the zero mode become infinitely large, while in the limit of $\alpha$ approaching unity, they all acquire the common value of the zero-mode threshold.

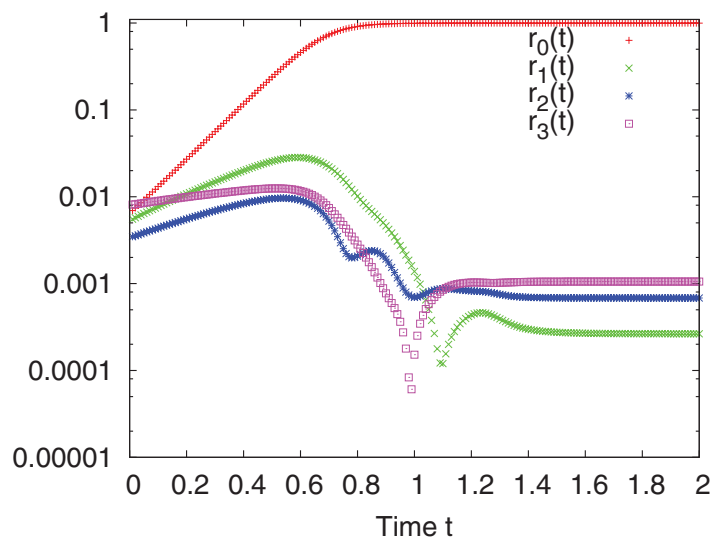

FIG. 2. (Color online) Time evolution of the observables $r_{0}(t), r_{1}(t), r_{2}(t)$, and $r_{3}(t)$ for a single realization of the initial state $\left\{\theta_{i}(0), \omega_{i}(0) ; i=1,2, \ldots, N\right\}$, where $\theta_{i}$ are chosen uniformly in $[-\pi, \pi]$, while $\omega_{i}$ are chosen from a Gaussian distribution with zero mean and unit variance [Eq. (29)]. Thus, initially, the system is in an unsynchronized state. Here $\alpha=0.5$ and $K=15$. For this value of $\alpha$, Eqs. (26) and (29) give $K_{c}^{(0)} \approx 1.59577, K_{c}^{(1)} \approx 4.26696, K_{c}^{(2)} \approx$ $6.53664, K_{c}^{(3)} \approx 7.71516, \ldots$, so that the Fourier modes $0,1,2,3$ are all linearly unstable. Consequently, $r_{0}(t), r_{1}(t), r_{2}(t)$, and $r_{3}(t)$ all grow in time from their initial values. The data in the plot are obtained from numerical simulations with $N=2^{14}$. 

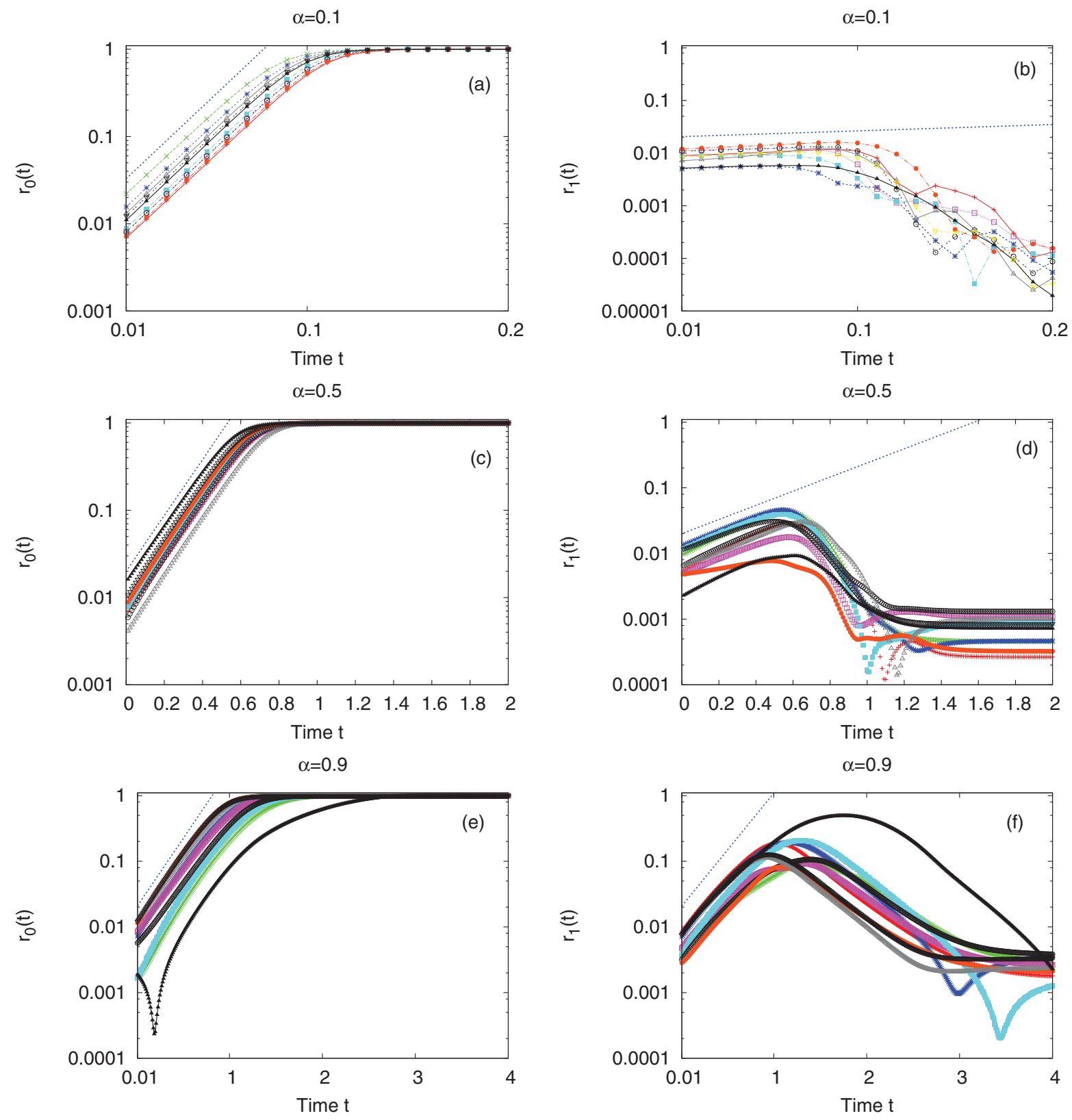

FIG. 3. (Color online) Time evolution of $r_{0}(t)$ and $r_{1}(t)$ for 10 different realizations of the initial state $\left\{\theta_{i}(0), \omega_{i}(0) ; i=1,2, \ldots, N\right\}$, where $\theta_{i}$ are chosen uniformly in $[-\pi, \pi]$, while $\omega_{i}$ are chosen from a Gaussian distribution with zero mean and unit variance [Eq. (29)]. Thus, initially, the system is in an unsynchronized state. Each figure corresponds to a value of $\alpha$ indicated therein. For $\alpha=0.1$, we took $K=100$. Equations $(26)$ and $(29)$ give $K_{c}^{(1)} \approx 25.89784, K_{c}^{(2)} \approx 59.98356, K_{c}^{(3)} \approx 76.10483, \ldots$ For $\alpha=0.5$, we took $K=15$; here $K_{c}^{(1)} \approx$ $4.26696, K_{c}^{(2)} \approx 6.53664, K_{c}^{(3)} \approx 7.71516, \ldots$. For $\alpha=0.9$, we took $K=10$; here $K_{c}^{(1)} \approx 1.88898, K_{c}^{(2)} \approx 2.04629, K_{c}^{(3)} \approx 2.12267, \ldots$. We have $K_{c}^{(0)} \approx 1.59577$, independent of $\alpha$. Thus, for these three values of $\alpha$ with the corresponding values of $K$, the Fourier modes 0 and 1 are linearly unstable. As a result, in all cases, $r_{0}(t)$ and $r_{1}(t)$ are expected to grow in time from their initial values as $r_{0}(t) \sim e^{\lambda_{0} t}, r_{1}(t) \sim e^{\lambda_{1} t}$. The data in the plots are obtained from numerical simulations with $N=2^{14}$. The dotted blue line in each plot shows the exponential growth with the rates $\lambda_{0}$ and $\lambda_{1}$ given by Eq. (30).

In simulations, we monitor the time evolution of the observable

$$
r_{m}(t)=\frac{1}{N}\left|\sum_{j=1}^{N} e^{i\left(\theta_{j}+2 \pi j m / N\right)}\right| ; \quad m=0,1,2, \ldots .
$$

Note that $r_{0}(t)$ corresponds to the case in which there is no spatial dependence of the oscillator phases and, thus, characterizes the mean-field mode.
From our analysis in the preceding section, we see that for $K>K_{c}^{(m)}$, when the $m$ th spatial mode of fluctuations is linearly unstable, $r_{m}(t)$ should grow exponentially in time: $r_{m}(t) \sim e^{\lambda_{m} t}$. In our simulations, we found that for large enough $K$, the observable $r_{0}(t)$ grows in time and saturates to a value of $O(1)$, while all the other $r_{m}(t)$, after showing an initial growth in time, decay to a value close to zero. An illustration of such a behavior is shown in Fig. 2. This observation implies 


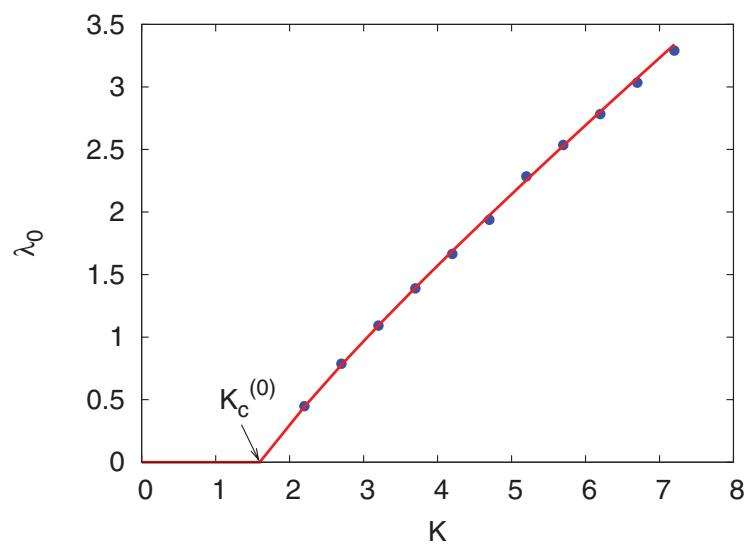

FIG. 4. (Color online) The points denote the values of the growth rate $\lambda_{0}$ obtained by fitting the growth of $r_{0}(t)$ in time as $r_{0}(t) \sim$ $e^{\lambda_{0} t}$ for short times, while starting from an unsynchronized state with Gaussian $g(\omega)$ [Eq. (29)]. The data for $r_{0}(t)$ were obtained by performing numerical simulations with $\alpha=0.5, N=2^{14}$, and by averaging over 100 initial realizations. The numerical errors are of the size of the points. The solid line shows the theoretical curve for $\lambda_{0}$ given by Eq. (30). From our theoretical analysis, the growth rate is zero for values of $K$ below $K_{c}^{(0)}$ and is nonzero above. In the figure, the theoretical $K_{c}^{(0)}$ is indicated.

that in the long-time limit, the dynamics is dominated by the mean-field mode, which drives the system to a synchronized state signaled by $r_{0}(t)$ settling to a value of order 1 .

In Fig. 3 we show the temporal evolution of $r_{0}(t)$ and $r_{1}(t)$ for 10 different realizations of the initial state for three different values of $\alpha$, namely, a value close to zero, a value close to unity, and a value in between. In each case, the value of $K$ is such that the Fourier modes 0 and 1 are linearly unstable; thus, $K$ is at least larger than $K_{c}^{(1)}$. Consequently, $r_{0}(t)$ and $r_{1}(t)$ are expected to grow in time from their initial values as $r_{0}(t) \sim e^{\lambda_{0} t}, r_{1}(t) \sim e^{\lambda_{1} t}$.

One may see from the plots for $r_{0}(t)$ in Fig. 3 that almost all realizations exhibit growth rates close to the theoretical value. It is then natural to average $r_{0}(t)$ over large enough number of initial realizations in order to reduce statistical fluctuations and extract the growth rate $\lambda_{0}$. We perform this exercise for $\alpha=0.5$ and display the result for $\lambda_{0}$ as a function of $K$ in Fig. 4, which shows a very good agreement with the theoretical prediction.

From the plots of $r_{1}(t)$ in Fig. 3, one may note that the growth rates for most initial realizations are close to the theoretical value, although there are some realizations for which significant deviations are seen. On decreasing the value of $K$ towards $K_{c}^{(1)}$, the number of this latter class of realizations increases, most significantly when $K$ is close to $K_{c}^{(1)}$. We believe that this could be due to pronounced finite- $N$ effects close to $K_{c}^{(1)}$. Also, note from Eq. (30) that $\lambda_{1}$ decreases with decreasing $K$. Since $r_{1}(t)$ in the limit of long times decays to a close-to-zero value, it leaves with a very narrow time window for the growth of $r_{1}(t)$.

From the plots in Fig. 3, one may observe that the growth rate of $r_{1}(t)$ goes to zero when $\alpha$ approaches zero, while it gets close to the rate for $r_{0}(t)$ when $\alpha$ approaches unity. This is in accordance with our discussion above on the behavior of thresholds as $\alpha$ approaches zero and unity.

\section{CONCLUDING REMARKS}

In this paper we considered a system of coupled phase-only oscillators, in which each site of a one-dimensional periodic lattice of $N$ sites contains one oscillator of distributed natural frequency. The oscillators are coupled through a power-law interaction $\sim(K / \widetilde{N}) r^{-\alpha}$ in their separation $r$ along the lattice length, where the exponent $\alpha$ lies in the range $0 \leqslant \alpha<1$, and $\widetilde{N}$ is a size-dependent normalization. The oscillator frequencies are taken to be distributed according to a density $g(\omega)$ that is symmetric about its mean (taken to be 0 ) and nonincreasing on $[0, \infty)$.

We studied the model in the continuum limit $N \rightarrow \infty$. In this limit one may define a local density of oscillators which evolves in time according to the continuity equation expressing the conservation of number of oscillators of each frequency under the dynamics. The unsynchronized state, uniform both in phase and in spatial coordinate, represents a stationary solution of the continuity equation. By analyzing the linear stability of such a state, we showed that when it is unstable, different spatial Fourier modes of fluctuations have different stability thresholds beyond which they grow exponentially in time with different rates. However, numerical simulations show that at long times, all the nonzero Fourier modes decay in time, while the zero mode (the "mean-field" mode) grows in time to dominate the instability process and drive the system to a synchronized state. Such a long-time dominance of the mean-field mode is known for systems with Hamiltonian systems [19]. The present work illustrates the dominance for dissipative dynamics within the ambit of a simple model, while leaving the proof of its general validity as an interesting open issue.

\section{ACKNOWLEDGMENTS}

We acknowledge useful discussions with Romain Bachelard and Thierry Dauxois, and financial support from INFN and ANR-10-CEXC-010-01, Chaire d'Excellence project. We are especially grateful to Alessandro Campa for many fruitful discussions, valuable inputs, and a careful reading of the manuscript. We thank Arkady Pikovsky for pointing out Ref. [16]. S.G. thanks Anandamohan Ghosh for directing him to the Kuramoto model.

\section{APPENDIX: A FAST NUMERICAL ALGORITHM TO COMPUTE THE INTERACTION TERM IN EQ. (2)}

Here we discuss a numerical algorithm that transforms the equations of motion (2) into a convenient form in which the interaction term is efficiently computed by a fast Fourier transform (FFT) scheme in times of order $N \ln N$ for a system of $N$ oscillators. Use of FFT requires that we choose a power of 2 for $N$.

We start with Eq. (2) which we rewrite as

$$
\frac{d \theta_{i}}{d t}=\omega_{i}+\frac{K}{\widetilde{N}} \sum_{j=1}^{N} \frac{\sin \left(\theta_{j}-\theta_{i}\right)}{\left(d_{i j}\right)^{\alpha}}
$$

where $d_{i j}$ is the shortest distance between sites $i$ and $j$ on a periodic lattice of $N$ sites. We set $d_{i i}=0$, while $d_{i j}$ for $i \neq j$ 
is given by

$$
d_{i j}=\left\{\begin{array}{cc}
|j-i| & \text { if } 1 \leqslant|j-i| \leqslant N / 2, \\
N-|j-i| & \text { otherwise. }
\end{array}\right.
$$

Equation (A1) may be rewritten as

$$
\frac{d \theta_{i}}{d t}=\omega_{i}+\frac{K}{\widetilde{N}}\left(\cos \theta_{i} \sum_{j=1}^{N} V_{i j} \sin \theta_{j}-\sin \theta_{i} \sum_{j=1}^{N} V_{i j} \cos \theta_{j}\right) .
$$

Here the first summation term may be interpreted as the $i$ th element of the $N \times 1$ column vector formed by the product of an $N \times N$ matrix $V=\left[V_{i j}\right]_{i, j=1,2, \ldots, N}$ with an $N \times 1$ column vector $\left(\sin \theta_{1} \sin \theta_{2} \ldots \sin \theta_{N}\right)^{T}$, where $V_{i j}=1 /\left(d_{i j}\right)^{\alpha}$. Similarly, the second summation term may be interpreted as the $i$ th element of the $N \times 1$ column vector formed by the product of $V$ with an $N \times 1$ column vector $\left(\cos \theta_{1} \cos \theta_{2} \ldots \cos \theta_{N}\right)^{T}$.

Writing out the matrix $V$, we see that it has the form

$$
V=\left[\begin{array}{ccccc}
v_{1} & v_{N} & \ldots & v_{3} & v_{2} \\
v_{2} & v_{1} & v_{N} & & v_{3} \\
\vdots & v_{2} & v_{1} & \ddots & \vdots \\
v_{N-1} & & \ddots & \ddots & v_{N} \\
v_{N} & v_{N-1} & \ldots & v_{2} & v_{1}
\end{array}\right],
$$

where $v_{1}=0$ and

$$
v_{r}=\left\{\begin{array}{cc}
1 /(r-1)^{\alpha} & \text { if } 2 \leqslant r \leqslant N / 2+1, \\
1 /(N-r+1)^{\alpha} & \text { otherwise. }
\end{array}\right.
$$

Thus, $V$ is a circulant matrix fully specified by the elements in the first column. The remaining columns of $V$ are each cyclic permutations of the elements in the first column, with offset equal to the column index.

Now, note that $V$ can be written as

$$
V=v_{1} I+v_{2} P+v_{3} P^{2}+\cdots+v_{N} P^{N-1},
$$

where $P$ is the $N \times N$ cyclic permutation matrix,

$$
\left[\begin{array}{ccccc}
0 & 0 & \cdots & 0 & 1 \\
1 & 0 & \cdots & 0 & 0 \\
0 & \ddots & \ddots & \vdots & \vdots \\
\vdots & \ddots & \ddots & 0 & 0 \\
0 & \cdots & 0 & 1 & 0
\end{array}\right]
$$

Since $P^{N}=I$, the $N \times N$ identity matrix, the eigenvalues of $P$ are given by $w_{j}=e^{i 2 \pi(j-1) / N} ; j=1,2, \ldots, N$, where $w_{j}$ is the $N$ th root of unity. Equation (A6) then implies that the eigenvalues of $V$ are given by $\Lambda_{j}=\sum_{k=1}^{N} v_{k} w_{j}^{k-1}$ for $j=1,2, \ldots, N$.

One may easily check that the eigenvectors of $V$ are the columns of the $N \times N$ unitary discrete Fourier transform matrix $F=\frac{1}{\sqrt{N}}\left[f_{j k}\right]_{j, k=1,2, \ldots, N}$, where

$$
f_{j k}=e^{-i 2 \pi(j-1)(k-1) / N} \quad \text { for } 1 \leqslant j, k \leqslant N .
$$

Then, one has $F^{-1} V F=\left[\Lambda_{j} \delta_{i j}\right]_{i, j=1,2, \ldots, N}$.

In terms of the matrices $F$ and $F^{-1}$, one may easily rewrite Eq. (A3) as

$$
\begin{aligned}
\frac{d \theta_{i}}{d t}= & \omega_{i}+\frac{K}{\widetilde{N}}\left[\cos \theta_{i} \sum_{j=1}^{N}\left(F^{-1}\right)_{i j} \Lambda_{j}(F \sin \theta)_{j}\right. \\
& \left.-\sin \theta_{i} \sum_{j=1}^{N}\left(F^{-1}\right)_{i j} \Lambda_{j}(F \cos \theta)_{j}\right],
\end{aligned}
$$

where $(F \sin \theta)_{j}$ [respectively, $\left.(F \cos \theta)_{j}\right]$ is the $j$ th element of the $N \times 1$ column vector formed by multiplying the matrix $F$ with the column vector $\left(\sin \theta_{1} \sin \theta_{2} \ldots \sin \theta_{N}\right)^{T}$ [respectively, $\left.\left(\cos \theta_{1} \cos \theta_{2} \ldots \cos \theta_{N}\right)^{T}\right]$. Note that $(F \sin \theta)_{j}$ and $(F \cos \theta)_{j}$ are just discrete Fourier transforms and may be computed very efficiently by standard FFT codes (see, e.g., Ref. [22]). The simulations reported in Sec. III were performed by integrating Eq. (A9).
[1] A. K. Ghosh, B. Chance, and E. K. Pye, Arch. Biochem. Biophys. 145, 319 (1971).

[2] J. Aldridge and E. K. Pye, Nature (London) 259, 670 (1976).

[3] C. S. Peskin, Mathematical Aspects of Heart Physiology (Courant Institute of Mathematical Science Publication, New York, 1975).

[4] D. C. Michaels, E. P. Matyas, and J. Jalife, Circulation Res. 61, 704 (1987).

[5] J. Buck and E. Buck, Sci. Am. 234, 74 (1976).

[6] J. Buck, Quart. Rev. Biol. 63, 265 (1988).

[7] K. Wiesenfeld, P. Colet, and S. H. Strogatz, Phys. Rev. Lett. 76, 404 (1996).

[8] K. Wiesenfeld, P. Colet, and S. H. Strogatz, Phys. Rev. E 57, 1563 (1998).

[9] A. Pikovsky, Synchronization: A Universal Concept in Nonlinear Sciences (Cambridge University Press, Cambridge, 2003).

[10] Y. Kuramoto, Chemical Oscillations, Waves and Turbulence (Springer, Berlin, 1984).
[11] S. H. Strogatz, Physica D 143, 1 (2000).

[12] J. A. Acebrón, L. L. Bonilla, C. J. Pérez Vicente, F. Ritort, and R. Spigler, Rev. Mod. Phys. 77, 137 (2005).

[13] P. König, A. K. Engel, and W. Singer, Proc. Natl. Acad. Sci. USA 92, 290 (1995)

[14] J. L. Rogers and L. T. Wille, Phys. Rev. E 54, R2193 (1996).

[15] M. S. O. Massunaga and M. Bahiana, Physica D 168, 136 (2002).

[16] A. Pikovsky and M. Rosenblum, Physica D 240, 872 (2011).

[17] D. Chowdhury and M. C. Cross, Phys. Rev. E 82, 016205 (2010).

[18] M. Maródi, F. d'Ovidio, and T. Vicsek, Phys. Rev. E 66, 011109 (2002).

[19] R. Bachelard, T. Dauxois, G. De Ninno, S. Ruffo, and F. Staniscia, Phys. Rev. E 83, 061132 (2011).

[20] A. Campa (private communication).

[21] R. E. Mirollo and S. H. Strogatz, J. Stat. Phys. 60, 245 (1990).

[22] H. M. Antia, Numerical Methods for Scientists and Engineers, 2nd ed. (Birkhauser, Basel, 2002). 